\title{
Incidence and modes of onset of early reinitiation of atrial fibrillation after successful internal cardioversion, and its prevention by intravenous sotalol
}

\author{
H-F Tse, C-P Lau, G M Ayers
}

\begin{abstract}
Objectives-To study the incidence and mode of onset of early reinitiation of atrial fibrillation (ERAF) following successful internal cardioversion of chronic atrial fibrillation, and to determine the effects of sotalol in the prevention of ERAF.

Design-The incidence and modes of onset of ERAF and the acute effects of intravenous sotalol in the prevention of ERAF were studied retrospectively.

Setting-Electrophysiology laboratory at a university teaching hospital.

Patients-64 patients, mean (SD) age 62 (10) years, who underwent internal cardioversion of chronic atrial fibrillation (mean duration of atrial fibrillation 31 (39) months).
\end{abstract}

Main outcome measures-ECGs and intracardiac electrograms recorded during the internal cardioversion of atrial fibrillation using $3 / 3 \mathrm{~ms}$ biphasic, $R$ wave synchronised shocks.

Results-52 patients (81\%) had successful electrical cardioversion, and $20(31 \%)$ of these had ERAF during the procedure. There was no clinical predictor for the occurrence of ERAF. Fifty eight episodes of ERAF were observed. Five ERAF episodes (9\%) had preceding bradycardia and 53 (91\%) of these were triggered by atrial premature beats with normal preceding heart rate. Atrial premature beats that reinitiated atrial fibrillation had a shorter coupling interval (333 (43) ms $v 396$ (100), $\mathrm{p}<0.001)$ and a lower prematurity index $(0.44(0.11) v 0.55(0.14), \mathrm{p}<0.001)$ than those that did not reinitiate atrial fibrillation. Repeated shock delivery and increasing the defibrillation energy did not prevent ERAF. Intravenous sotalol infusion decreased the numbers of atrial premature beats and prolonged their coupling interval, and prevented ERAF after repeated defibrillation in $83 \%$ of patients with ERAF. Conclusions-ERAF is a significant clinical problem after successful internal cardioversion of chronic atrial fibrillation, and was observed in up to $31 \%$ of patients. In most episodes, ERAF was triggered by short coupling atrial premature beats with preceding normal heart rate. Intravenous sotalol was effective in preventing ERAF in most cases. (Heart 1999;82:319-324)

Keywords: atrial fibrillation; low energy cardioversion; sotalol
Low energy internal cardioversion has been shown to be very effective in converting atrial fibrillation. ${ }^{1-6}$ This procedure requires only mild sedation and is more effective than external cardioversion. ${ }^{78}$ Thus the use of low energy internal cardioversion may result in restoration of sinus rhythm in a greater number of patients. However, unstable sinus rhythm followed by early reinitiation of atrial fibrillation (ERAF) shortly after successful electrical cardioversion was observed in up to $13-36 \%$ of patients. ${ }^{9-11}$ It could significantly limit the number of patients with successful clinical termination of atrial fibrillation. A rational approach to preventing ERAF may depend on a better understanding of its incidence and modes of onset. Furthermore, the role of class III antiarrhythmic agents in preventing the spontaneous reinitiation of atrial fibrillation after successful cardioversion remains largely unknown. The purpose of the present study was to investigate the incidence and clinical variables that predict the occurrence of ERAF shortly after successful internal cardioversion of chronic atrial fibrillation, the modes of onset of ERAF, and the efficacy of a class III agent, sotalol, in the prevention of ERAF.

\section{Methods}

PATIENTS

We carried out a retrospective study of 64 consecutive patients with chronic atrial fibrillation of at least one month's duration who underwent internal cardioversion. A detail clinical examination was performed and a complete medical history was taken. Routine 12 lead ECG, 24 hour Holter monitoring, chest $x$ ray, routine laboratory and thyroid tests, and transthoracic and transoesophageal echocardiography were assessed in all patients. Patients with the following conditions were excluded from study: reversible causes of atrial fibrillation, moderate to severe valvar heart disease, unstable angina or recent myocardial infarction, class III or IV heart failure, contraindication to sotalol (asthma, chronic obstructive pulmonary disease, significant sinus nodal or conduction system dysfunction, or a previous adverse reaction to a $\beta$ blocker), or evidence of left atrial thrombus. No study patient had been receiving any class I, III, or IV antiarrhythmic drugs for more than five half lives, or amiodarone for longer than three months.

All patients gave written informed consent before the procedure, and the protocol was approved by the ethics committee of the University of Hong Kong. 
ANTICOAGULATION PROTOCOL

All patients were treated with oral anticoagulants, using warfarin to achieve an international normalised ratio (INR) of 2-3 for at least three weeks before the procedure. Warfarin was discontinued two days before the procedure and was replaced with an intravenous heparin infusion. The heparin infusion was stopped four hours before and restarted after the procedure.

The INR was checked daily and immediately before the procedure, and the minimum INR value considered safe for venous puncture was less than 1.5. Warfarin was restarted after the procedure and heparin infusion was discontinued when the therapeutic INR of $2-3$ was achieved.

STUDY PROTOCOL

Details of the internal cardioversion procedure in our laboratory have been described previously. ${ }^{3}$ In brief, low energy biatrial shocks were delivered through two transvenously introduced defibrillation catheters. One of these catheters was positioned in the coronary sinus and one in the anterolateral right atrium. In 36 patients, a pair of temporary $6 \mathrm{~F}$ decapolar catheters (Elecath, New Jersey, USA) was used. In the remaining 28 patients, two custom built spring coil electrodes were used (Perimeter 7107 and 7023, InControl Inc, Redmond, Washington, USA). The right atrial catheter served as the cathode and the coronary sinus catheter as the anode. Two additional $6 \mathrm{~F}$ catheters were positioned, one in the right ventricular apex (for shock synchronisation and postshock pacing) and one in high right atrium (for recording the atrial electrogram and pacing); both were advanced from the right femoral vein. The defibrillation catheters were connected to a custom made external atrial defibrillator (XAD, InControl Inc) capable of delivering an $\mathrm{R}$ wave synchronised biphasic shock waveform $(3 / 3 \mathrm{~ms})$ with a leading edge voltage that could be programmed between 10 and $400 \mathrm{~V}$.

Patients were sedated with intravenous midazolam $(0.05 \mathrm{mg} / \mathrm{kg})$ and pethidine $(0.5$ $\mathrm{mg} / \mathrm{kg}$ ), and additional doses were given as required. Starting with a $20 \mathrm{~V}$ test shock, R wave synchronised shocks were delivered starting at $180 \mathrm{~V}$ and increasing in steps of $40 \mathrm{~V}$ until sinus rhythm was restored, or the until two at the highest output of $400 \mathrm{~V}$ had been delivered. Between unsuccessful defibrillation attempts, at least two minutes were allowed to elapse before the next shock was applied.

During the study, two surface ECGs (lead II and V6), a bipolar high right atrium and ventricular electrogram, and arterial blood pressure were recorded simultaneously. ERAF was defined as the occurrence of spontaneous reinitiation of a sustained atrial fibrillation episode after successful internal cardioversion. Following successful internal cardioversion, the rhythm was monitored for up to five minutes to look for any episodes of ERAF and their mode of onset. Where ERAF were triggered by atrial premature beats, their characteristics were studied and compared with those not triggered by atrial premature beats. We determined the following:

- coupling interval of atrial premature beats (atrial to atrial premature beats)

- mean atrial cycle length of three to five consecutive preceding beats

- the prematurity index (atrial premature beat cycle length/preceding rhythm cycle length) using a high right atrium endocardial electrogram

- the density of atrial premature beats (number of atrial premature beats/min).

In those patients with ERAF, a repeat defibrillation shock at the initially successful energy was given. If the atrial fibrillation still recurred, up to two high energy shocks at the maximum voltage of $400 \mathrm{~V}$ were tested in some patients. For patients who still had ERAF, an intravenous infusion of sotalol $(1.5 \mathrm{mg} / \mathrm{kg})$ over 30 minutes was given, and defibrillation shocks were repeated using the same step up protocol, starting at $180 \mathrm{~V}$. In those patients who failed cardioversion at baseline, intravenous sotalol was also given to determine whether it could reduce the atrial defibrillation requirement. The effect of sotalol on the atrial defibrillation threshold has been reported before. ${ }^{12}$ Oral sotalol $160-320 \mathrm{mg} /$ day was given to those patients who had successful cardioversion and were maintained in sinus rhythm after the procedure.

\section{STATISTICAL METHODS}

Continuous data are presented as mean (SD). Statistical analysis was performed by analysis of variance, Mann-Whitney U test, or Fisher exact test, as appropriate, and between group comparisons were performed by a multiple Bonferroni test. A probability (p) value of $<0.05$ was considered statistically significant.

\section{Results}

PATIENT CHARACTERISTICS

The 64 patients included 45 men and 19 women, aged 36 to 80 years, mean 62 (10) years. The duration of atrial fibrillation ranged from 1 to 192 months, mean 31 (39). Mean left ventricular ejection fraction was 58 (10)\% (range $35 \%$ to $75 \%$ ) and mean left atrial diameter was $4.4(0.6) \mathrm{cm}$ (range 3.1 to $5.5 \mathrm{~cm}$ ). Underlying heart disease was present in 36 patients (56\%), including hypertension (23), treated thyroid heart disease (5), mild valvar heart disease (4), dilated cardiomyopathy (3), and congenital heart disease (1).

\section{EFFICACY OF INTERNAL CARDIOVERSION AND} INCIDENCE OF ERAF

These results are summarised in fig 1. Before sotalol, successful internal cardioversion of atrial fibrillation was achieved in 52 patients $(81 \%)$ at a mean threshold of $325(60) \mathrm{V}$ and a mean energy of 3.92 (1.4) J. However, 20 patients $(31 \%)$ had ERAF shortly after successful cardioversion and failed to maintain stable sinus rhythm. Two patients had only one 


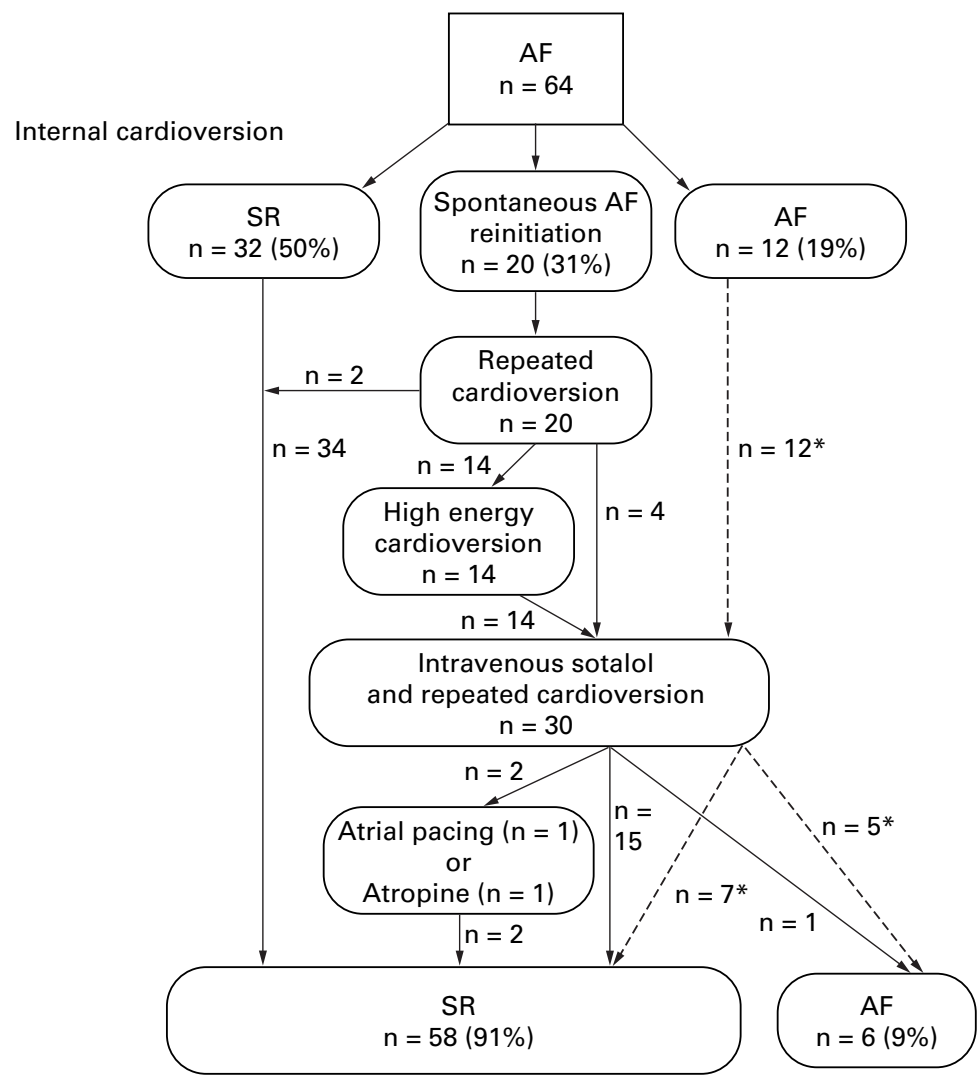

Figure 1 Flow chart showing the outcome of patients after low energy internal cardioversion of atrial fibrillation $(A F)$ and the results of treatment for spontaneous reinitiation of atrial fibrillation by repeated defibrillation, intravenous sotalol and atropine, and atrial pacing. SR, sinus rhythm.

episode of ERAF and remained in stable sinus rhythm after repeated shock delivery. In the remaining 18 patients with repeated episodes of ERAF, increasing the shock energy to maximum output of $400 \mathrm{~V}$ was tested in 14 patients but failed to prevent recurrence of atrial fibrillation in any of them. Twelve patients (19\%) had unsuccessful cardioversion despite two defibrillation shocks at maximum output of $400 \mathrm{~V}$. The clinical characteristics of these patients are given in table 1 . There were no significant differences between the patients with and without reinitiation of ERAF, and those in whom sinus rhythm could not be restored, with respect to age, sex ratio, left ventricular ejection fraction, left atrial size, use of $\beta$ blockers or digitalis, and absence of underlying heart

Table 1 Clinical characteristics of study patients according to the result of internal cardioversion

\begin{tabular}{llll}
\hline & $\begin{array}{l}\text { Successful } \\
\text { cardioversion }\end{array}$ & $\begin{array}{l}\text { Early reinitiation of } \\
\text { atrial fibrillation }\end{array}$ & $\begin{array}{l}\text { Failed } \\
\text { cardioversion }\end{array}$ \\
\hline Number (\%) & $32(50)$ & $20(31)$ & $12(19)$ \\
Age (years) & $61(11)$ & $64(9)$ & $65(9)$ \\
Sex (M:F) & $20: 12$ & $15: 5$ & $10: 2$ \\
Left atrial diameter (mm) & $44(6)$ & $43(7)$ & $44(7)$ \\
Left ventricular ejection fraction & $59(12)$ & $58(8)$ & $57(12)$ \\
Threshold (V) & $324(56)$ & $326(54)$ & - \\
Energy (J) & $3.95(1.24)$ & $4.06(1.45)$ & - \\
Drug treatment (n) & 12 & 8 & 6 \\
$\quad \beta$ Blocker & 7 & 2 & 2 \\
$\quad$ Digitalis & 13 & 10 & 4 \\
$\quad$ None & 14 & 9 & 5 \\
No heart disease (n) & $25(37)^{\star}$ & $28(44)^{\star}$ & $51(31)$ \\
Duration of atrial fibrillation (months) & & & \\
\hline
\end{tabular}

Values are mean $(\mathrm{SD})$

${ }^{\star} \mathrm{p}<0.05 v$ failed cardioversion. disease. Patients with stable sinus rhythm and ERAF had a comparable duration of atrial fibrillation, whereas patients with unsuccessful cardioversion had a significantly longer duration of atrial fibrillation $(p<0.05)$.

MODE OF ONSET OF ERAF

Before drug testing, 58 episodes of spontaneous atrial fibrillation reinitiation were observed at a mean duration of 10 (4) seconds after internal cardioversion. Five episodes of ERAF $(9 \%)$, in two patients, were initiated after a period of bradycardia (with a mean atrial cycle length > $1200 \mathrm{~ms}$ ) (fig 2A). In $53 \mathrm{ERAF}$ episodes $(91 \%)$, atrial fibrillation was reinitiated by atrial premature beats after a short period of organised electrical activity without preceding bradycardia (fig $2 \mathrm{~B}$ ). In 19 of these ERAF episodes in five patients, the episodes were preceded by a long-short sequence of their preceding atrial rhythm cycle length (defined where the shorter cycle length is $<75 \%$ of the longer one) (fig 2C). Furthermore, in 16 ERAF episodes in six patients, the atrial premature beats that reinitiated the atrial fibrillation seemed to be repetitive in nature: these atrial premature beats had a similar P wave morphology and a consistent cycle length with less than $10 \mathrm{~ms}$ variability, suggesting a similar origin for all.

In patients with ERAF, atrial premature beats without triggering atrial fibrillation were also observed. There was no significant difference in the preceding atrial cycle length between atrial premature beats associated with or not associated with reinitiation of atrial fibrillation. Atrial premature beats that resulted in reinitiation of atrial fibrillation had a shorter beat to beat coupling interval (333 (43) ms $v$ $396(100) \mathrm{ms}, \mathrm{p}<0.001)$ and greater prematurity with a lower prematurity index than those that did not result in reinitiation of atrial fibrillation $(0.44(0.11) v 0.55(0.14), \mathrm{p}<0.001)$.

\section{EFFICACY OF INTRAVENOUS SOTALOL IN}

PREVENTING ERAF

An intravenous sotalol infusion (at a mean dose of $124(32) \mathrm{mg}$ ) was given to 18 patients who had persistent ERAF despite repeated shock delivery. None of them was converted to sinus rhythm after sotalol administration, and repeated internal cardioversion was performed. There was no significant change in the atrial defibrillation requirement after sotalol administration compared with baseline (327 (56) $v$ 310 (24) V, p > 0.05). Sotalol was effective in preventing reinitiation of atrial fibrillation in 15 patients $(83 \%)$ and in maintaining stable sinus rhythm. After sotalol infusion, the density of atrial premature beats decreased significantly compared with baseline, at 4.4 (3.8) $v 14.7$ (11) atrial premature beats/min, $\mathrm{p}<0.0001$. Furthermore, the cycle length of the preceding atrial rhythm (1055 (155) ms $v 801$ (207) ms, $\mathrm{p}<0.001)$ and the atrial premature beat coupling interval (401 (54) ms v 333 (43) ms, $\mathrm{p}<0.001)$ were also prolonged after sotalol infusion compared with baseline. In those patients who failed cardioversion at baseline, 

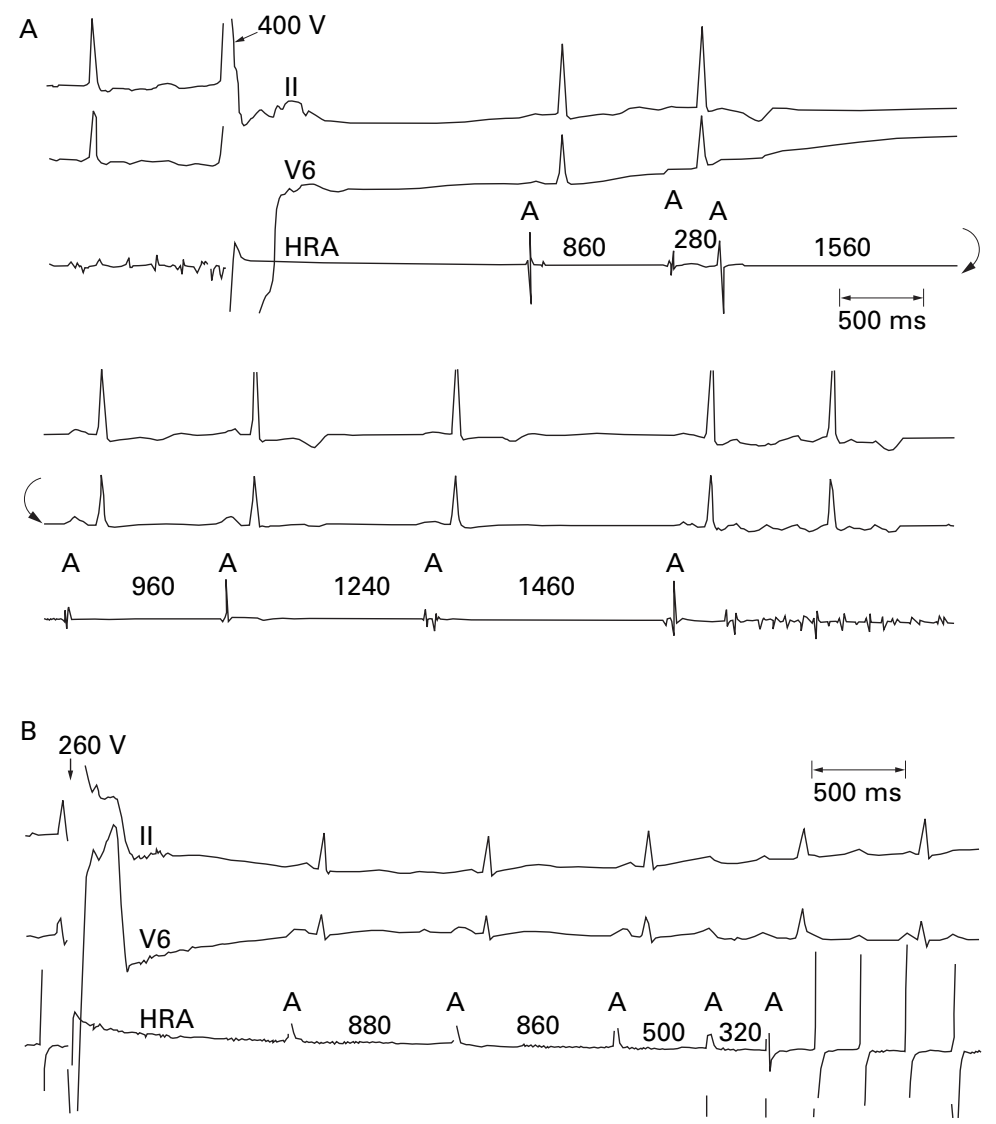

C
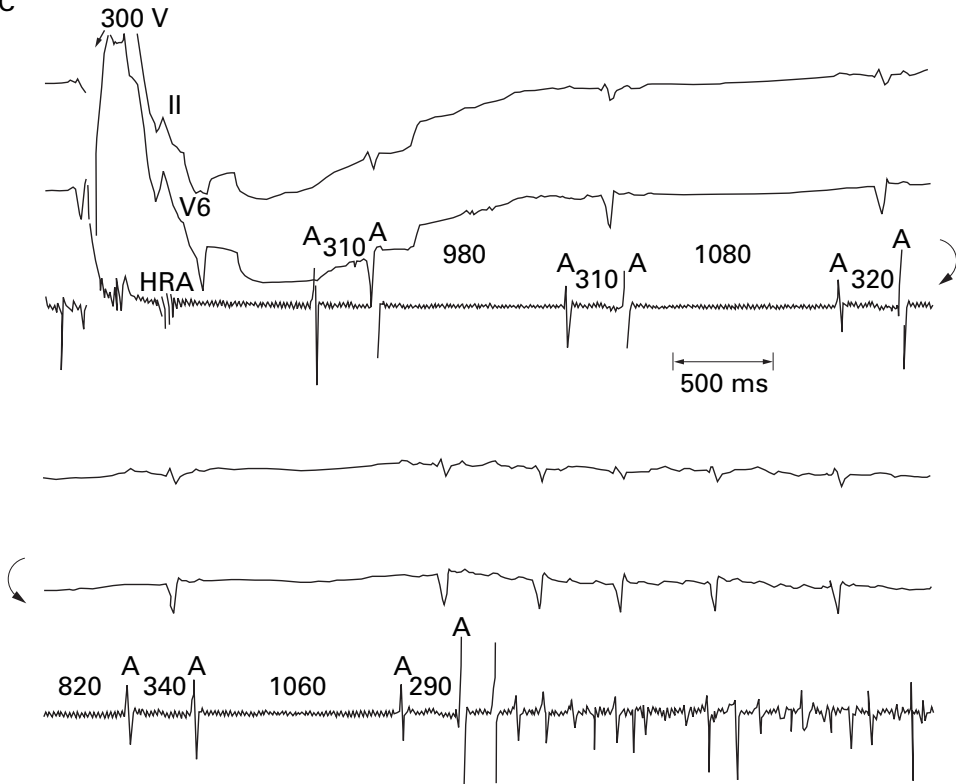

Figure 2 Different modes of onset of early reinitiation of atrial fibrillation $(A F)$. (A) $A F$ reinitiation preceding by bradycardia (mean atrial cycle length $=1240 \mathrm{~ms}$ ). (B) $A F$ was reinitiated an atrial premature beat $(A P B)$ after a short period of organised electrical activity. Note the normal heart rate preceding the onset of $A P B$. (C) AF was reinitiated by $A P B$ with preceding long/short cycles. HRA, high right atrium.

seven of 12 had successful cardioversion after sotalol infusion and none of these had ERAF.

In the two patients with ERAF associated with bradycardia, sotalol alone was not effective in preventing recurrence of atrial fibrillation but worsened the bradycardia, with long sinus pauses after cardioversion. Elimination of this bradycardia after cardioversion seemed to be effective in preventing further ERAF. Thus the administration of intravenous atropine before repeated defibrillation resulted in sinus rhythm without sinus pauses or ERAF in one of the patients. In the other patient, atrial pacing at a cycle length of $500 \mathrm{~ms}$ was started immediately after defibrillation and was effective in preventing recurrence of further atrial fibrillation. Both of these patients remained in stable sinus rhythm thereafter. One patient failed to maintain in sinus rhythm owing to persistent ERAF, despite administration of sotalol and repeated defibrillation (fig 1 ).

COMPLICATIONS

Overall, $1402 \mathrm{R}$ wave synchronised shocks were delivered to the 64 patients without ventricular proarrhythmia or acute complication. Cardiac enzymes including creatine kinase and its isoenzyme CK-MB measured before and after defibrillation did not show any significant change. One patient developed symptomatic persistent junctional bradycardia after sotalol which required temporary pacing for two days. Two patients experienced heart failure with oral sotalol and required drug termination.

\section{Discussion}

EFFICACY OF INTERNAL CARDIOVERSION AND INCIDENCE OF ERAF

Restoration of sinus rhythm is the preferred goal of treatment in patients with persistent atrial fibrillation, as it should eliminate symptoms, improve exercise capacity, and reduce thromboembolic complications, and as it might improve survival. However, for patients with atrial fibrillation of prolonged duration, electrical cardioversion with external shocks requires higher energies and has a low success rate. ${ }^{13}$ Recently, internal cardioversion has been shown to be highly effective in conversion of atrial fibrillation, even where external cardioversion has failed. ${ }^{1-8}$ The results of our study have confirmed the efficacy and safety of low energy internal cardioversion in restoring sinus rhythm, with a success rate of $81 \%$ in patients with prolonged atrial fibrillation before cardioversion (mean duration 31 (39) months).

Recurrences of atrial fibrillation are commonly observed after successful cardioversion. In previous studies, unstable sinus rhythm followed by ERAF recurrence occurred in $13-36 \%$ of patients within minutes after successful electrical cardioversion, either external or internal. ${ }^{9-11}$ In the present study we showed that up to $31 \%$ of patients with chronic atrial fibrillation without pretreatment with antiarrhythmic agents had spontaneous ERAF shortly after cardioversion. We found that the occurrence of ERAF was not related to the previous arrhythmia duration or to any of the clinical variables (age, sex ratio, left atrial size, left ventricular ejection fraction, defibrillation energy and voltage, use of $\beta$ blockers and digitalis, or the type of heart disease). Repeated cardioversion was only effective at preventing ERAF in $10 \%$ of patients, and ERAF could not 
be prevented by increasing the energy of the defibrillation shock.

MODES OF ONSET OF ERAF

It is well known that a spontaneous atrial fibrillation episode is usually initiated by atrial premature beats. ${ }^{14}{ }^{15}$ Previous studies using Holter recording have shown that atrial premature beats that initiated atrial fibrillation had a shorter coupling interval and greater prematurity than isolated atrial premature beats that did not initiate atrial fibrillation. ${ }^{16-18}$ Consistent with these findings, recent studies ${ }^{10}{ }^{11}$ have demonstrated that ERAF following internal cardioversion was usually preceded by an atrial premature beat with a short coupling interval, and occurred within the first minutes after cardioversion. However, the characteristics of cardiac rhythm preceding the onset of ERAF remain unclear. Observations on the heart rate, initiation sequence, and coupling interval of the initiating atrial premature beats may allow treatment strategies to be tailored appropriately. Suppression of ERAF has important implications for the use of temporary low energy internal cardioversion and may require more frequent treatment with an implantable atrial defibrillator. ${ }^{19}$

In the present study, the onset of ERAF episodes and the characteristics of the atrial premature beats were recorded using a surface ECG and an intra-atrial electrogram. In about $10 \%$ of patients, the ERAF episodes were preceded by a long sinus pause. The cause of the bradycardia may be underlying sinoatrial disease or electrical remodelling of the sinus node by atrial fibrillation. ${ }^{20}$ It may be further aggravated by shock induced sinus node suppression. ${ }^{3}$ During bradycardia, the escape atrial ectopic activity, together with the pause dependent conduction abnormalities, may trigger the onset of atrial fibrillation. ${ }^{18}$ However, in the majority of episodes (90\%), atrial fibrillation was reinitiated by atrial premature beats with a short coupling interval which were not preceded by bradycardia or tachycardia. Consistent with previous studies, ${ }^{10}{ }^{11} 16-18$ atrial premature beats that reinitiated atrial fibrillation had a significantly shorter cycle length and greater prematurity than those that did not. We observed that in up to $30 \%$ of patients the onset of atrial fibrillation was preceded by a long-short atrial sequence. A recent study has shown the occurrence of a preceding longshort atrial sequence was strongly associated with development of atrial fibrillation even in patients without a history of this arrhythmia. ${ }^{21}$ It has been postulated that long-short cardiac sequences increase the dispersion of myocardial refractoriness and their vulnerability for reentrant arrhythmias. ${ }^{22}{ }^{23}$ Furthermore, in one third of our patients, the atrial premature beats that initiated atrial fibrillation had a very similar P wave morphology and consistent cycle length, suggesting the presence of a similar or focal origin. These patients might have a focal source of atrial fibrillation, and more detailed endocardial mapping may be useful to identify the origin of these atrial premature beats. ${ }^{23}$
EFFECTS OF INTRAVENOUS SOTALOL

Previous studies ${ }^{9-11}$ suggested that administering antiarrhythmic drugs before cardioversion might prevent early recurrence of atrial fibrillation. Limited data show that intravenous flecainide may acutely suppress ERAF ${ }^{10}$ However, the role of class III antiarrhythmic drugs in the acute suppression of ERAF remains unclear. In the present study, we observed that intravenous sotalol could suppress ERAF acutely in the majority of patients (83\%). Furthermore, of those patients who initially failed cardioversion and had successful cardioversion after sotalol infusion, none had ERAF. This also suggested that pretreatment with sotalol before cardioversion could prevent ERAF. Although the mechanism of action of sotalol in suppressing reinitiation of atrial fibrillation remains unclear, our results suggest that it might prevent ERAF by suppressing the onset of atrial premature beats (probably by its $\beta$ blocking effect) and prolonging the coupling interval of atrial premature beats (possibly a class III effect). Both decreasing the numbers of atrial premature beats and prolonging their coupling interval may reduce the likelihood that such beats will encounter the vulnerable atrial period and this will help prevent reinitiation of atrial fibrillation. However, in those patients with ERAF preceding by bradycardia, sotalol caused further worsening of the bradycardia and could not prevent reinitiation of atrial fibrillation.

\section{CLINICAL IMPLICATIONS}

A rational approach to preventing ERAF may depend on a careful evaluation of the pattern of onset. Although our data suggest that intravenous sotalol can effectively suppress ERAF acutely in the majority of patients, other non-pharmacological treatments may also be useful in preventing ERAF. Thus in patients with bradycardia dependent onset of atrial fibrillation, atrial support pacing may reduce the occurrence of atrial fibrillation. ${ }^{24}$ As shown in the present study, the prevention of bradycardia or sinus pauses by atrial pacing or atropine was effective at preventing these atrial fibrillation episodes. Furthermore, whether atrial pacing prevents ERAF by eliminating the long-short rhythm cycle that precedes the onset of atrial fibrillation or by suppressing the atrial premature beats which might precipitate atrial fibrillation requires further study. In a subset of patients, the atrial fibrillation episodes were reinitiated by atrial premature beats with a consistent coupling interval and similar P wave morphology, suggesting the presence of a focal source of atrial fibrillation. This type of focal atrial fibrillation may be amenable to radiofrequency ablation of the focus, as shown in recent studies. ${ }^{25} 26$

\section{STUDY LIMITATIONS}

Owing to the retrospective nature of the study and the fact that only two surface ECGs and a high right atrial electrogram were recorded during the procedure, the origin of the atrial premature beats that reinitiated the atrial fibrillation could not be determined. More 
detailed mapping of the onset of atrial fibrillation and the origin of the atrial premature beats, using multiple endocardial catheters or new mapping techniques, might provide a better understanding of the mechanisms of ERAF. Although our observations suggested that sotalol might be effective in preventing ERAF, it is impossible to draw firm conclusions because the study was not randomised. Nevertheless, before sotalol infusion all patients with ERAF underwent repeated cardioversions that were followed by reinitiation of atrial fibrillation. This suggests that the observed effect after sotalol administration was attributable to its use. Furthermore, our observations that sotalol suppressed atrial premature beats and that none of the patients in whom cardioversion was successful only after sotalol infusion had ERAF provide further indirect evidence to support the efficacy of sotalol in preventing ERAF.

CONCLUSIONS

We have shown that ERAF occurred in up to $31 \%$ of patients with chronic atrial fibrillation after successful internal cardioversion. This is a significant clinical problem which limits the number of patients having successful clinical termination of sustained atrial fibrillation. It has implications for the use of both temporary and permanent transvenous defibrillation in treating atrial fibrillation. ERAF is usually preceded by the occurrence of atrial premature beats, although the cardiac sequences preceding their onset seem to be heterogeneous. Intravenous sotalol effectively suppressed ERAF in the majority of patients. The use of other antiarrhythmic agents and the role of non-pharmacological treatment for the prevention of ERAF after internal cardioversion require further investigation. This study was supported by research grant HKU, account code
338/041/0020, from the University and Polytechnic Grant $338 / 041 / 0020$, from the University and Polytechnic Grant
Council, Hong Kong and InControl Inc, Redmond, Washington, USA.

1 Kumagai K, Yamanouchi Y, Hiroki T, et al. Effects of transcatheter cardioversion on chronic lone atrial fibrillation. catheter cardioversion

2 Murgatroyd FD, Slade AK, Sopher SM, et al. Efficacy and tolerability of transvenous low energy cardioversion of paroxysmal atrial fibrillation in humans. $f \mathrm{Am}$ Coll Cardio 1995;25:1347-53.

3 Lok NS, Lau CP, Ho DSW, et al. Hemodynamic effects and clinical determinants of defibrillation threshold for low energy transvenous atrial defibrillation in patients with chronic atrial fibrillation. PACE 1997;20:899-908.
4 Levy S, Richard P, Lau CP, et al. Multicenter low energy transvenous atrial defibrillation (XAD) trial results in
different subsets of atrial fibrillation. $7 \mathrm{Am}$ Coll Cardiol different subsets

5 Levy S, Richard P, Gueunoun M, et al. Low-energy cardioversion of spontaneous atrial fibrillation. Immediate and long term results. Circulation 1997;96:253-9.

6 Alt E, Schmitt C, Ammer R, et al. Initial experience with intracardiac atrial defibrillation in patients with chronic atrial fibrillation. PACE 1994;17:1067-78.

7 Levy S, Lauribe P, Dolla E, et al. A randomized comparison of external and internal cardioversion of chronic atrial fibrillation. Circulation 1992;86:1415-20.

8 Schmitt C, Alt E, Plewan A, et al. Low energy intracardiac cardioversion after failed conventional external cardioversion of atrial fibrillation. F Am Coll Cardiol 1996;28:994-9.

9 Bianconi L, Mennuni M, Lukic V, et al. Effects of oral propafenone administration before electrical cardioversion of chronic atrial fibrillation: a placebo-controlled study. $\mathcal{F}$ Am Coll Cardiol 1996;28:700-6.

10 Timmermans C, Rodriguez LM, Smeets JLRM, et al. Immediate reinitiation of atrial fibrillation following internal atrial defibrillation. $\mathcal{F}$ Cardiovasc Electrophysiol 1998;9:122-8.

11 Sra J, Biehl M, Blanck Z, et al. Spontaneous reinitiation of atrial fibrillation following transvenous atrial defibrillation. PACE 1998;21:1105-10.

12 Lau CP, Lok NS. A comparison of transvenous atrial defibrillation of acute and chronic atrial fibrillation and the effect of intravenous sotalol on human atrial defibrillation threshold. PACE 1997;20:1-11.

13 Lown B. Electrical cardioversion of cardiac arrhythmias. $\mathrm{Br}$ Heart f 1967;29:469-87.

14 Bennett MA, Pentecost BL. The pattern of onset and spontaneous cessation of atrial fibrillation in man. Circulation 1970;41:981-8.

15 Kennedy RJ, Killip T. The onset of atrial fibrillation in man. Am Heart f 1971;82:429-31.

16 Capucci A, Santarelli A, Boriani G, et al. Atrial premature beats coupling interval determines lone paroxysmal atrial fibrillation onset. Int $\mathcal{F}$ Cardiol 1992;36:87-93.

17 Mehra R, Hill M. Prevention of atrial fibrillation/flutter by pacing techniques. In: Saksena S, Luderitz B, eds. Interventional electrophysiology. A textbook. New York: Futura Publishing Co, 1996:521-40.

18 Murgatroyd F. Mode of onset of spontaneous episodes of atrial fibrillation: implications for the prevention of atrial fibrillation by pacing. In: Daubert JC, Prystowsky EN, fibrillation by pacing. In: Daubert JC, Prystowsky EN, Ripart A, eds. Prevention of tachyarrhythmias with cardiac
pacing. New York: Futura Publishing Co, 1997:53-65.

19 Wellen HJJ, Lau CP, Luderitz B, et al, for the Metrix Investigators. Atrioverter, an implantable device for the treatment of atrial fibrillation. Circulation 1998;98:1651-6.

20 Elvan A, Zipes DP. Pacing induced chronic atrial fibrillation impairs sinus node function in dogs. Circulation 1996;94: 1600-6.

21 Strickberger SA, Man KC, Daoud EG, et al. Adenosineinduced atrial arrhythmia: a prospective analysis. Ann Intern Med 1997;127:417-22.

22 Kay GN, Plumb VJ, Arciniegas JG, et al. Torsade de pointes: the long-short initiating sequence and other clinical features: observations in 32 patients. $\mathcal{F} \mathrm{Am}$ Coll Cardiol 1983;2:806-17.

23 Denker S, Lehmann MH, Mahmud R, et al. Facilitation of macroreentry within the His-Purkinje system with abrupt changes in cycle length. Circulation 1984;69:26-32.

24 Attuel P, Pellerin D, Mugica J, et al. DDD pacing: an effective treatment modality for recurrent atrial arrhythmias. tive treatment modality
PACE $1988 ; 88: 1647-54$

25 Lau CP, Tse HF, Ayers GM. Defibrillation guided radiofrequency ablation of atrial fibrillation secondary to an atrial focus. F Am Coll Cardiol 1998;33:1217-26.

26 Jais $\mathrm{P}$, Haissaquerre M, Shah DC, et al. A focal source of atrial fibrillation treated by discrete radiofrequency ablation. Circulation 1997;95:572-6. 